\title{
nature
}

genetics

\section{Risky business}

\section{Recent discoveries and improvements in technology have boosted the profile of 'personalized genomics'. But the demonstrated complexities of the genetics of common disease suggest caution-and an urgent need for more data, both in and out of the lab.}

W e compile a weekly press release to alert journalists to papers in Nature Genetics that may be of general interest. Arguably the most widely discussed paper in the last two years was the report by Yoshiura et al. describing a SNP in ABCC11 as the determinant of human earwax type. Science writers certainly had a good time with this one ("Finally," wrote one wag at USA Today). This paper is likely just one of many that will appear in the journal addressing the genetics of visible human traits. Earlier this year a paper by Weedon et al. reported a variant in HMGA2 to be associated with height. And on page 1443 of this issue, Patrick Sulem and colleagues present genome-wide association scans for variants influencing hair, eye and skin pigmentation in Europeans. These data, in concert with other recent findings, provide the fullest picture so far of the genetic architecture of variation in the most visible of human traits.

That there should be such interest in the genetic basis of these traits is unsurprising. The reasons why we look like our parents are at the heart of the way genetics is first introduced to schoolchildren and get at notions of family and kinship that transcend science. The attention paid to these traits also provides an opportunity to educate a larger public about the complexity of nonmendelian genetics. It was, after all, human stature that R.A. Fisher used as the primary example in his landmark 1918 paper that essentially founded the field of quantitative genetics. And in general, the popular coverage of the work by Weedon et al. was helpful, headlines reporting a 'height gene' or a 'tall gene' notwithstanding. Indeed, most accounts noted that stature in humans is a polygenic trait.

The need for both physicians and their patients to be better educated about complex genetics has taken on added urgency of late. Here are just a few stories in recent months in the area of 'personalized genomics': the publication of the sequence of Craig Venter's genome; the announcement of the sequencing of Jim Watson's genome and that of a Han Chinese individual; the release of an extensive report by the US Department of Health and Human Services entitled "Personalized Health Care: Opportunities, Pathways, Resources"; the announcement by deCODE Genetics of a test for type 2 diabetes risk as assessed by TCF7L2 genotype; and Nature's coverage of the remarkable story of Hugh Rienhoff, a physician who is personally organizing the sequencing of candidate genes from his daughter's genome in an effort to understand her illness.

There are also companies, among them Genovations, 23andme and Navigenics, that will offer genotyping and consulting services to individuals who want to learn more about their potential risks for common diseases. Despite all of the progress that's been made, however, one has to wonder whether robust risk assessments can now be offered to individu- als and whether this information will make a difference in their lives. This seems especially true given how little we know about rare variants that may have a major role in determining an individual's risk for a disease. With the possible exception of age-related macular degeneration, how much can we say with confidence about the spectrum of risk?

The website for Navigenics features one of its officers declaring: "The clinical information that comes back to the consumer is presented in a way so that the confusion is removed." But the literature on the genetics of common disease is not exactly crystal clear, even to insiders. For example, the paper reporting the sequence and analysis of Craig Venter's genome had this gem buried deep in the results section: "We have also been able to detect inconsistencies between detected genotypes in the donor's DNA and the expected phenotype based on the literature given the known phenotype of the HuRef donor. For example, the donor's LCT genotype should confer adult lactose tolerance according to published literature, but this does not match with the self-reported phenotype of the donor's lactose intolerance." No harm done in this case, as we suspect Dr. Venter is still on a milkshake-free diet. But the consequences for the assessment of risk for more serious disorders may not be so easily shrugged off.

To be sure, there are knowledgeable researchers associated with these companies, and the use of genetic data by individuals and their doctors is entirely compatible with the vision of the founders of the Human Genome Project. But what will consumers do with the sort of provisional information that can be offered to them? Fortunately, the National Human Genome Research Institute is in the midst of the Multiplex Initiative (http://www.multiplex.nih.gov), a crossdisciplinary project led by Colleen McBride, Lawrence Brody and Andy Baxevanis. The goal is to assess the responses of at least 1,000 individuals to genetic testing for 15 variants robustly associated with 8 common conditions or diseases. These variants were selected because they are relatively common and because some kind of preventive behavior is available to lower the risk of getting the disease. Participants will take web-based surveys to find out whether they want to be tested, and also to find out what they make of the results they are given if they decide to go ahead. Conversations with educators will be offered. The information on the study's website regarding the impact of the variants is admirably clear and low-key, including this important admonition: "As we learn more about these genes, the risk information we have may change." McBride and colleagues emphasize that this is a genuine experiment in behavioral science, with no particular expectation as to the outcome. The results bear watching, and they may help physicians and genetic counselors craft sound, evidence-based applications of personalized genomics in the future. 